\title{
A rare case: Penetrating craneoencefalic trauma by knife
}

\begin{abstract}
Background: Cranioencephalic penetrating trauma (CPT) is caused by a sharp or shortpointed object that passes through the bone, dura mater, brain and other structures. Its incidence is unknown and few cases are described; penetrating injuries represent $0.4 \%$, therefore there is no protocolized management.
\end{abstract}

Case report: A 24-year-old male patient suffered penetrating trauma at left parietal region with a "knife"; he was sutured and sent home with analgesics. Five days after the trauma, he was admitted for headache, disorientation and decreased visual acuity. X-ray (XR) of Cranium evidencing a foreign body, therefore it is sent to a reference hospital.

Evolution: The diagnosis is confirmed by a computerized tomography (CT) scan of the skull with 3-dimensional reconstruction (3D) plus CT angiography (angio CT), which shows "knife" in the left parietal region without vascular compromise. Neurosurgeons perform removal of the foreign body plus a dura mater plasty. Patient stay 12 days hospitalized with a favorable evolution and improvement of neurological symptomatology.

Conclusion: CPT due to a knife is an emergency and there is no protocolized management. The removal of the foreign body must be done in a hospital for the risk of lesions of large vessels.

Keywords: craniocerebral trauma, post-head injury, gun violence, knife, craniotomy, cerebral hemorrhage
Volume II Issue 6 - 202I

\author{
Galo Fabián García Ordóñez, Andrea Priscila \\ Guillermo Cornejo, Luis Fernando García \\ Ordóñez, Danny Renán García Ordóñez, \\ Jenner Quilson Aguilar Castillo, Jorge \\ Rodrigo Villacis Sandoval \\ Neurosurgery Service,Vicente Corral Moscoso Hospital, \\ Ecuador
}

\begin{abstract}
Correspondence: Galo Fabián García Ordóñez, Genera Surgeon. Neurosurgery Service, Vicente Corral Moscoso Hospital, 01 0204, Cuenca, Azuay, Ecuador, Email galogarciao@hotmail.com
\end{abstract}

Received:September 26, 2021 | Published: December 13, 2021

\section{Introduction}

The purpose of this article is to review the definition, epidemiology, clinic, diagnosis and treatment for penetrating cranioencephalic trauma and retention of foreign bodies. The CPT is an emergency because it could put in risk the life of the patient, is caused for a sharp or cut-conduntent object (metal, wood, plastic, other); by an aggressor, self-inflicted or in accidental way. The object impacted with a considerable force traverse the bone of skull, dura mater, brain and other structures depending of the enter point; this can be retained days, months, years or has an exit point. ${ }^{1-3}$

The incidence is unknown and only described a few cases in the literature; about $90 \%$ of CPT are produced in men, more than $2 / 3$ are localized in the left side (right-handed aggressors), being the more vulnerable zones: frontoparietal, orbital and temporal region (low thickness of temporal bone and the short distance of neurovascular structures of vital importance). The penetrating injuries represent around $0,4 \%$ (bullet wounds $4,6 \%$ ). Currently there is not management protocolized because this low incidence. It cans happen unnoticed and had retention of fragments of the object, if this associated to injuries on the scalp. The injuries depend of the localization and extension, presenting as: focal deficit, edema, intracranial hypertension, hemorrhages, pneumocephalus, intraventricular hemorrhage, skull fractures, wound site infections, neuroinfections, sepsis, septic shock and death. ${ }^{1,3,4-6}$

The best diagnostic method is the CT scan of skull that allows to determine the journey and the extension of the injury. However, the metal strange body produces artifacts and it does not allow to correctly assess neurovascular injuries. The CT with reconstruction 3D could to ease the diagnosis and the presurgical planning. In the case of non-metallic objects like "wood", the use of magnetic resonance is recommended, because it allows to differentiate around the tissue, however it is not recommended rutinary use because it consumes time, low accessibility, also is inadequate for the bones and can be dangerous in ferromagnetic objects. If suspected of arteriovenous injuries or traumatic aneurysms, you should make a brain angio CT, the strange bodies can produce vasospasm or stenosis, therefore, there must be a high diagnostic suspect to correlate with the antecedent. ${ }^{1,4,7-12}$

The treatment and removal of the foreign body should be individualized for each case, the main objective should be the removal in a hospital environment and not in the trauma site because there may be a compromise of large vessels that generates fatal hemorrhages, it must be performed, here the need to perform a pre-surgical angio CT, in addition aggressive antibiotic treatment should be administered. In case of identifying vascular lesions, protection of the damaged arteries should be ensured by preoperative endovascular occlusion (to prevent intraoperative bleeding) or intraoperative artery control; on the other hand, if there are no vascular lesions, foreign bodies could be eliminated directly. Organic foreign bodies, such as wood or bamboo, are carriers and the best environment for infections (brain abscess, meningitis and cerebritis), so they must be removed in their entirety, while foreign bodies metallic or bones fragments adjacent to important structures and difficult to extract, could be retained in the brain, because extraction can cause further damage. ${ }^{11-13}$

Among the complications of CPT are: posttraumatic epilepsy, vascular complications (5-40\% pseudo or true traumatic aneurysms, arteriovenous malformations and vasospasm), leakage of cerebrospinal fluid and infections. Infection rates associated with the foreign body are estimated from 6 to $21 \%$. The use of Antibiotic therapy will depend on the commitment of the dura mater; if this 
not compromising, the use of intravenous ceftriaxone for 7 days and then 10 days of oral ciprofloxacin is recommended; while in case of rupture, a triple scheme is recommended: ceftriaxone, vancomycin (staphylococcus aureus) and metronidazole for 14 days followed by 10 days of oral ciprofloxacin. ${ }^{14,15}$

The prognosis, severity and sequelae will depend on the early identification, location, depth, type of neurovascular damage and the value of Glasgow Coma Scale (GCS) at admission. ${ }^{16,17}$

\section{Case report}

\section{History and examination}

A 24-year-old male patient who, is admitted to a first level hospital in apparent ethylic state, suffered penetrating trauma at left parietal region with a "knife" of approximately $3 \mathrm{~cm}$; he was sutured and sent home with analgesics. However, patient reenters after 5 days for presented a high intensity holocranial headache, disorientation, and a reduction in visual acuity, due to a history of trauma, performing Lateral XR of Cranium, observing a foreign body with metal density compatible with a "Knife" (Figure 1A). This is transferred to a third level hospital for the neurosurgical service, in the neurological examination shows: disorientation, paraphasias, dyslalia, dysarthria, dyscalculia, left right dissociation, hemiparesis facio brachio crura with muscular strength $4 / 5$, isochoria, normoreactivity, decreased visual acuity, GSC 14/15, afebrile and hemodynamically stable.

The simple CT scan of the skull with $3 \mathrm{D}$ reconstruction and angio CT shows an image with metallic density at the level of the left parietal region, with 3D reconstruction confirms the presence of intracerebral "knife", without compromise of cerebral blood vessels (Figure 1B \& C).

\section{Operation}

The patient underwent a left parietal craniotomy with extirpation of a metallic material ("knife"), plus a dura mater plasty and closure of the skull. Uncomplicated procedure with approximate bleeding $300 \mathrm{cc}$. Surgical Findings: metallic object of 9 centimeters compatible with "knife handle", embedded at the level of parietal bone with dural and intraparenchymal involvement; was necessary to drill the bone around it and posterior craniectomy (Figure 1D \& F).
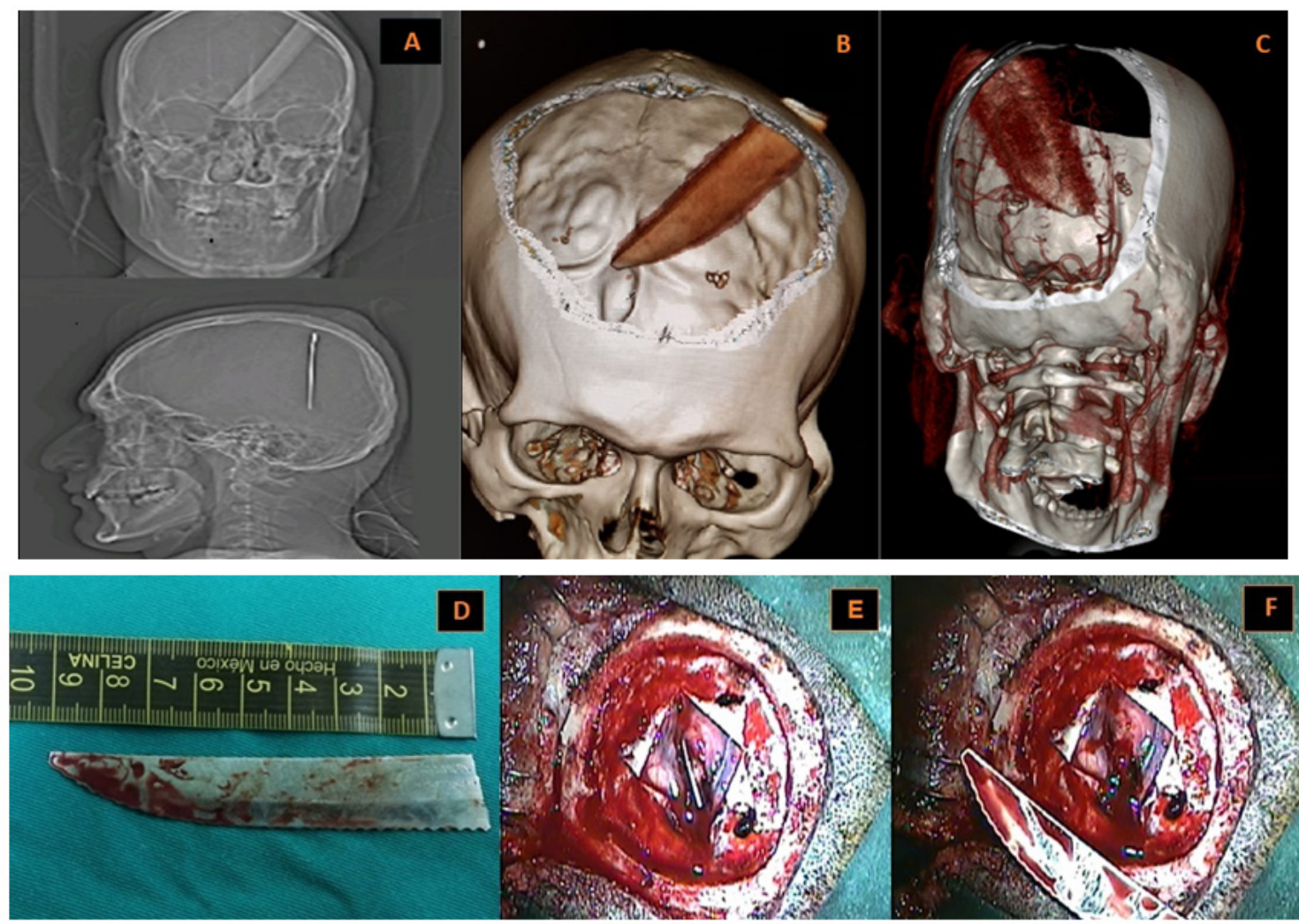

Figure I Lateral $X R$ of Cranium, 3D reconstruction and angio $C T$ iamges.

\section{Postoperative course}

Patient enters in ICU stays in mechanical ventilation for 2 days. Simple CT scan after surgery performed 24 and 48 hours, shows an intraparenchymal hemorrhage in the surgical site, perilesional edema and pneumocephalus (Figure 2A \& C).

Patient remains hospitalized for 12 days, 2 days in ICU, 10 days in neurosurgery service, the neurological examination presents: paraphasia, dislalia, right hemiparesis FM 4/5, right - left dissociation and decrease in bilateral visual acuity. Assessment by the ophthalmologist shows a normal fundus, indicating that the visual alteration is due to the traumatic antecedent.

Patient is discharged at day 12 with improvement of neurological symptoms, mild dysmetria ang GSC 15/15. CT scan was performed at post-surgical month, showing decreased intraparenchymal hemorrhage (Figure 2D-F). 


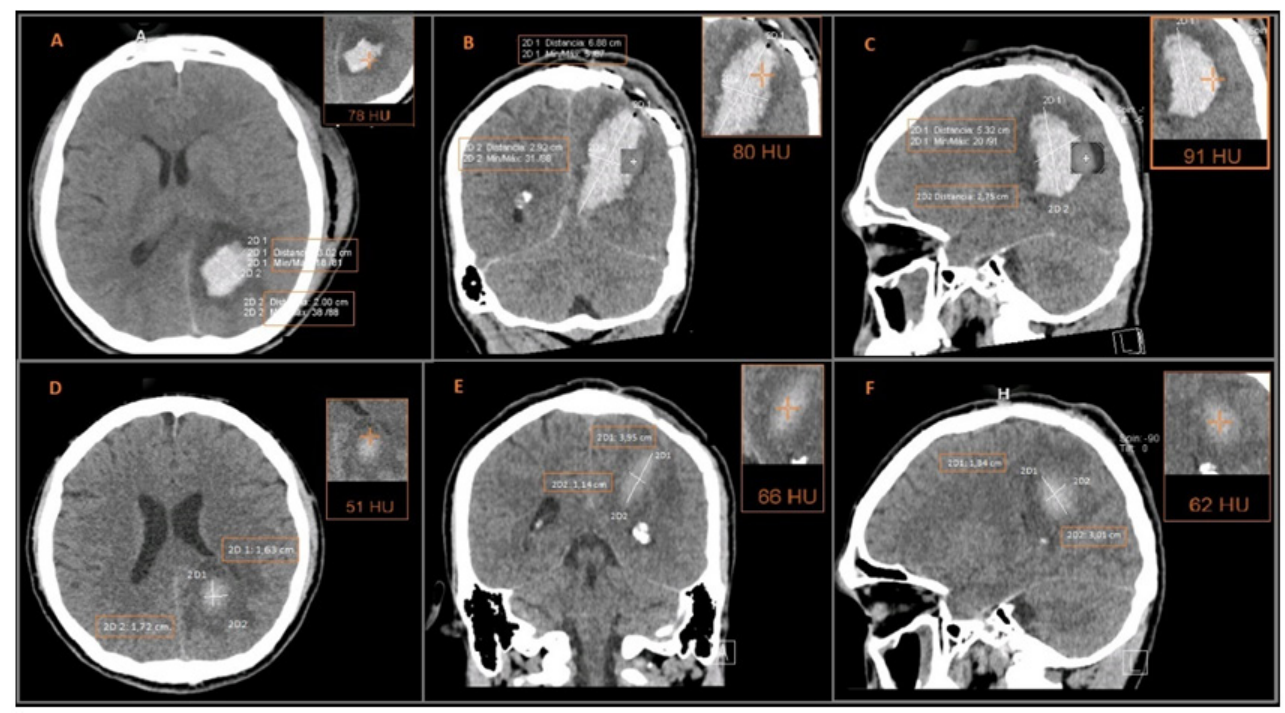

Figure 2 Intraparenchymal hemorrhage in the surgical site.

\section{Discussion}

Cranioencephalic penetrating injuries are rare $(0.4 \%)$, they are also known as penetrating injuries without missiles (impact velocity of $<100 \mathrm{~m} / \mathrm{s}$ ); caused by assaults, self-inflicted injuries (psychiatric patients) and accidents, which cause injuries by laceration and maceration, while projectiles cause injuries by kinetic and thermal energy. There are few cases described in the literature, so there is no protocolized and standardized management. The removal of the foreign body must be done in a hospital environment, due to the risk of neurovascular injuries such as: hemorrhages, irreversible brain injuries and death. In contrast to firearm injuries, penetrating injuries are associated with a more localized brain injury and therefore with a good postoperative result if handled properly. ${ }^{5,16,17}$

The severity of the CPT for stab injuries will depend on the type of weapon, location, depth and trajectory; this may become fatal as a result of massive cerebral hemorrhage and direct lesions of brain structures. ${ }^{18}$

In Latin America, the largest description cases of CPT were made in Nicaragua in a retrospective study that included 51 patients with stab injuries ("Machete"). Mostly men $(n=43)$, between 16 and 30 years, the majority affected the left frontoparietal region. The average length of the laceration was $10.41 \mathrm{~cm}(3-18 \mathrm{~cm})$, which compromised the dura mater $(4.55 \mathrm{~cm}) ; 77 \%$ of the lesions $(\mathrm{n}=17)$ were greater than $10 \mathrm{~cm} ; 8 \%(\mathrm{n}=4)$ presented lesions in the venous sinuses, all with the superior sagittal sinus. $82 \%$ of the patients $(n=42)$ presented an GSC $\geq 14,10 \%(\mathrm{n}=5)$ GSC $9-13$ and $8 \%(\mathrm{n}=4)$ GSC $\leq 8$. Neurological deficits were observed such as: hemiplegia or hemiparesis $(8 \%)$, aphasia $(6 \%)$, seizures $(4 \%)$ and a syncopal episode $(2 \%)$. Surgical criteria were: laceration of $10 \mathrm{~cm}(\mathrm{n}=20)$, open intracranial wound ( $\mathrm{n}$ $=8)$, pneumocephalus $(n=7)$, cerebral contusion $(n=6)$, intracranial hemorrhage $(\mathrm{n}=5)$ and depressed fracture $(\mathrm{n}=5)$. In addition, was performed dura mater plasty $(\mathrm{n}=9)$ and periosteal graft or the tensor fascia lata $(n=21)$ Postoperative complications were rare, such as: visible skull defect 12\% $(\mathrm{n}=6)$, infection $6 \%(\mathrm{n}=3)$ (all underwent surgical cleaning), neurological complications no specified $4 \%$ ( $n=$ $2)$ and mixed neurological complications $2 \%(n=1)$. At discharge, $92 \%$ of patients $(\mathrm{n}=47)$ had minimal disability, $6 \%(\mathrm{n}=3)$ moderate disability, and $2 \%(\mathrm{n}=1)$ with severe disability. The mortality was $0 \%{ }^{19}$
The risk of post-traumatic epilepsy (PTE) is high, probably due to a direct cortical lesion with subsequent scarring. Post-traumatic seizures can be immediate ( $<24$ hours), early ( $<1$ week) and late ( $>1$ week). The factors associated with PTE were: score GSC on admission, type of injury, transventricular injury, focal neurological deficit, infections, early onset seizures, posttraumatic amnesia, depressive fracture and dura mater penetration. The use of anticonvulsants is controversial, because reports with short-term follow-ups indicate a reduction in early seizures, but not late. ${ }^{20-22}$

The treatment should be based on the complete extraction of the foreign body and its fragments, if necessary (because its increased risk of infection), broad spectrum antibiotics and tetanus prophylaxis. Currently, the use of intraoperative CT scan in high level centers in developed countries is described, finding better surgical results. ${ }^{20,23}$

In our case, the patient underwent surgery 24 hours after admission, pre-surgical CT scan and angio CT were evaluated, without evidence of vascular injury. Craniotomy, foreign body extirpation, dura mater plasty and closure of the skull was performed without complications. The foreign body was completely extracted, broad-spectrum antibiotics were used (ceftriaxone and linezolid) and no anticonvulsants were used. He had a favorable neurological evolution, with paraphasia, dyslalia, dysmetria and diminished visual acuity.

The patient was followed for 6 months without presenting infection of the surgical site, cerebrospinal fluid fistula or early or late seizures. The neurological symptomatology was improving with the decrease and reabsorption of intracerebral hemorrhage, in addition to rehabilitation. The prognosis will depend on the GSC on admission, state of the pupils and the site of the lesion. A value of GSC $>8 \mathrm{y}<13$, with normal pupillary response, without ventricular compromise, will benefit from early surgical treatment. On the other hand, bilateral hemispheric lesion, multilobular or brainstem, transventricular lesion, mass effect and secondary projectiles far from the trajectory are unfavorable prognoses..$^{20,24-27}$

\section{Conclusion}

Cranioencephalic penetrating trauma with a knife is a surgical emergency, that may put the patient's life at risk depending on the affected area. 
The removal of the foreign body must be done in a hospital environment, because can involvement of large vessels. In addition, there must be a high diagnostic suspicion due to the history of the trauma.t. Currently, there is no protocolized management because had a low casuistry with which it is presented.

\section{Acknowledgments}

None.

\section{Conflicts of interest}

The authors report no conflict of interest concerning the materials or methods used in this study or the findings specified in this paper.

\section{References}

1. Yarandi KK, Jelodar S, Khalatbari MR, et al. Stab Wounds to the Head; Case Series, Review of Stab Wounds to the Head; Case Series, Review of Literature, and Proposed Management. Asian J Neurosurg . 2018;13(3):754-759.

2. El Sayeda M, Hassan Saad R, Fereir A. Undiagnosed impacted knife blade from a penetrative orbital injury: A case report. El Sayed M, Hassan Saad R, Fereir A. Undiagnosed impacted knife blade from a penetrative orbital injury: A case report. Int J Surg Case Rep. 2018;53:254-258.

3. LomelÍ JJ, Rivero A. Traumatismo penetrante de cráneo. Casoclínico. Rev Hosp Jua Mex. 2017;84(3):177-179.

4. Jáñez-García L, Mencía-Gutiérrez E, Gutiérrez-Díaz E, et al. Occult Orbital Injury with Dagger Fragment with Resulting Pneumocephalus. Case Rep Ophthalmol Med. 2018;5093417.

5. Yusuf AS, Mahmud MR, Alfin JD, et al. Clinical Presentation and Outcome of Impalement Craniocerebral Injuries - A CASE SERIES. $J$ West Afr Coll Surg. 2017;7(2):112-123.

6. Rosenfeld JV, Bell RS, Armonda R. Current concepts in penetrating and blast injury to the central nervous system. World J Surg. 2015;39(6):1352-1362.

7. Mashriqi F, Iwanaga J, Loukas M, et al. Penetrating Orbital Injuries: A Review. Cureus. 2017;9(9):e1725.

8. Lan ZG, Richard SA, Li J, et al. Nonprojectile penetrating iron rod from the oral cavity to the posterior cranial fossa: a case report and review of literature. Int Med Case Rep J. 2018;11:41-45.

9. Alvis-Miranda HR, M Rubiano A, Agrawal A, et al. Craniocerebral Gunshot Injuries; A Review of the Current Literature. Bull Emerg Trauma. 2016;4(2):65-74.

10. You B, Cheng Y, Zhang J, et al. Application of contrast-enhanced T1-weighted MRI-based 3D reconstruction of the dural tail sign in meningioma resection. J Neurosurg. 2016;125(1):46-52.

11. Zhang D, Chen J, Han K, et al. Management of Penetrating Skull Base Injury: A Single Institutional Experience and Review of the Literature. Biomed Res Int. 2017;2838167.
12. Kazim SF, Bhatti AU, Godil SS. Craniocerebral injury by penetration of a T-shaped metallic spanner: A rare presentation. Surg Neurol Int. 2013;4:2.

13. Zapata L, Wright EJ, Nakaji P. Clinical and Neurosurgical Management of Cranial Machete Injuries: The Experience of a Tertiary Referral Center in Nicaragua. World Neurosurg. 2018;116:140-143.

14. Enicker B, Madiba TE. Cranial injuries secondary to assault with a machete. Injury. 2014;45(9):1355-1358.

15. De Holanda LF, Pereira BJA, Holanda RR, et al. Neurosurgical Management of Nonmissile Penetrating Cranial Lesions. World Neurosurg. 2016;90:420-429.

16. Del Castillo-Calcáneo JD, Bravo-Angel U, Mendez-Olan R, et al. Traumatic brain injury with a machete penetrating the dura and brain: Case report from southeast Mexico. Int J Surg Case Rep. 2016;23:169-172.

17. Shi J, Mao Y, Cao J, et al. Management of screwdriver-induced penetrating brain injury: a case report. BMC Surg. 2017;17(1):3.

18. Alvis-Miranda HR, Adie Villafañe R, Rojas A, et al. Management of Craniocerebral Gunshot Injuries: A Review. Korean J Neurotrauma. 2015;11(2):35-43.

19. Guerrero-Domínguez R, Luengo-Pastor MÁ, Jimeno-Puche I, et al. [Trans-oral intracranial stab wound with airway invasion]. Med Intensiva. 2015;39(7):451-453.

20. Younes W. Craniocerebral Injuries by Impacted Foreign Objects: Case Series and Literature Review. Egyp J Neurosurg. 2015;30(1):55-62.

21. Kim TW, Shim YS, Oh SY, et al. Head Injury by Pneumatic Nail Gun: A Case Report. Korean J Neurotrauma. 2014;10(2):137-138.

22. Veiga $\mathrm{P}$, Mazon $\mathrm{P}$, Gonzalvez M, et al. Traumatismo penetrante facial porarpón. A propósito de uncaso. [Penetrating facial trauma by harpoon. About a case]. Rev Esp Cir Oral Maxilofac. 2015;37(1):27-31.

23. Estebanez G, Garavito D, López L, et al. Penetrating Orbital-Cranial Injuries Management in a Limited Resource Hospital in Latin America. Craniomaxillofac Trauma Reconstr. 2015;8(4):356-362.

24. Martínez-Bustamante D, Pérez-Cárdenas S, Ortiz-Nieto JM, et al. Heridas craneales por proyectil de arma de fuego en población civil: análisis de la experiencia de un centroen Monterrey, México [Craniocerebral gunshot wounds in civilian population: Analysis of experience in a single center in Monterrey, México]. Cir Cir. 2015;83(2):94-99.

25. Arslankoylu AE, Ozgur A, Komur M. Birdshots in brain. J Emerg Trauma Shock. 2012;5(4):370-371.

26. Weisbrod AB, Rodriguez C, Bell R, et al. Long-term outcomes of combat casualties sustaining penetrating traumatic brain injury. $J$ Trauma Acute Care Surg . 2012;73(6):1525-1530.

27. Kazim SF, Shamim MS, Tahir MZ, et al. Management of penetrating brain injury. J Emerg Trauma Shock. 2011;4(3):395-402. 